\title{
EVOLUÇÃO DO MOVIMENTO ROTACIONAL LIVRE DE TORQUES EXTERNOS NAS REGIÕES DE LIBRAÇÃO E CIRCULAÇÃO
}

\author{
M.A.R.ALMEIDA ${ }^{1}$, M.C.ZANARDI ${ }^{1}$, W. R. SILVA $^{2}$, R. E. S. CABETTE ${ }^{3}$ \\ 1. Faculdade de Engenharia de Guaratinguetá - UNESP \\ Caixa Postal 205 CEP 12.516-410 - Guaratinguetá, SP, BRASIL \\ E-mails: mari.matematica@yahoo.com.br, cecilia@feg.unesp.br
}

2. Instituto Nacional de Pesquisas Espaciais - INPE

Caixa Postal 515 CEP 12.227-010 -São José dos Campos, SP, BRASIL

E-mails: reis.williamegmail.com

\author{
3. Centro Universitário Salesiano de São Paulo - UNISAL \\ CEP 12.600 - 100, Lorena, SP, BRASIL \\ E-mails: recabette@uol.com.br
}

\begin{abstract}
In this paper, the rotational motion without external torques is analyzed in order to investigate the temporal behavior of the variables that describe the motion in the regions of libration and circulation around the equilibrium points. To describe the rotational motion of the satellite are used Andoyer canonical variables. Applications are made to satellite with distinct principal moments of inertia. Three equilibrium points are determined, two points are unstable and one steady. In the temporal behavior there are periodic variations in a angle variable and a metric variable and there is a linear variation for the angular variable directly related to the rotation of the satellite. The results obtained are important for the analysis of rotational motion when the external torques are included in the equations of motion.
\end{abstract}

Keywords - Celestial mechanics, rotational motion, andoyer variables, equilibrium points.

Resumo - O movimento rotacional livre de torques externos é aqui analisado com o objetivo de investigar o comportamento temporal das variáveis que descrevem o movimento nas regiões de libração e circulação ao redor dos pontos de equilíbrio. Para descrever o movimento rotacional do satélite são utilizadas as variáveis canônicas de Andoyer. Aplicações são realizadas para um satélite de médio porte com momentos principais de inércia distintos. São determinados 3 pontos de equilíbrio, sendo dois pontos instáveis e apenas um estável. No comportamento temporal verificam-se variações periódicas em uma variável angular e uma variável métrica e uma variação linear para a variável angular diretamente relacionada com a rotação do satélite. Os resultados obtidos são importantes para a continuidade da análise do movimento rotacional quando os torques externos são incluídos nas equações do movimento.

Palavras-chave — Mecânica Celeste, movimento rotacional, variáveis de Andoyer, pontos de equilíbrio.

\section{Introdução}

Este trabalho enfoca o movimento rotacional de um satélite artificial na ausência de torques externos, analisando as regiões de libração e circulação ao redor dos pontos de equilíbrio das equações do movimento. Aplicações são realizadas para um satélite de médio porte com momentos principais de inércia distintos. Para descrever o movimento rotacional do satélite são utilizadas as variáveis canônicas de Andoyer. Tais variáveis, além do fato de possuírem significado físico, são convenientes para aplicação de métodos de teoria de perturbação, quando se deseja fazer uma abordagem analítica ao movimento rotacional. Essas variáveis também são convenientes para a aplicação de métodos de estabilidade para sistemas Hamiltonianos.

Considerando os sistemas de coordenadas do satélite: sistema de eixos principais de inércia Oxyz (denominando sistema principal), sistema equatorial OXYZ (com eixos coincidentes com o equador terrestre, denominado sistema do satélite) e o sistema do momento angular $\mathbf{O} \mathbf{x}_{\mathbf{m}} \mathbf{y}_{\mathbf{m}} \mathbf{z}_{\mathbf{m}}$ (eixo $\mathbf{O} \mathbf{z}_{\mathbf{m}}$ coincide com a direção do momento angular de rotação do satélite $\mathbf{L}_{2}$ ).

As variáveis de Andoyer (Kinoshita, 1972) para descrever o movimento rotacional do satélite, são aqui definidas:

- As variáveis angulares $\ell_{1}, \ell_{2}$ e $\ell_{3}$ são ângulos que relacionam os diferentes sistemas de referência envolvidos e estão apresentadas na Figura 1;

- As variáveis métricas: é o módulo do vetor momento angular de rotação $\overrightarrow{L_{2}}, L$ é a projeção de $\overrightarrow{L_{2}}$ no eixo $Z$ no sistema principal ( $\left.L \quad J\right)$ e é a projeção de $\overrightarrow{L_{2}}$ no eixo equatorial $Z(L$ ) .

Os desenvolvimentos foram realizados com o auxilio dos softwares MATLAB e MATHEMATICA. 


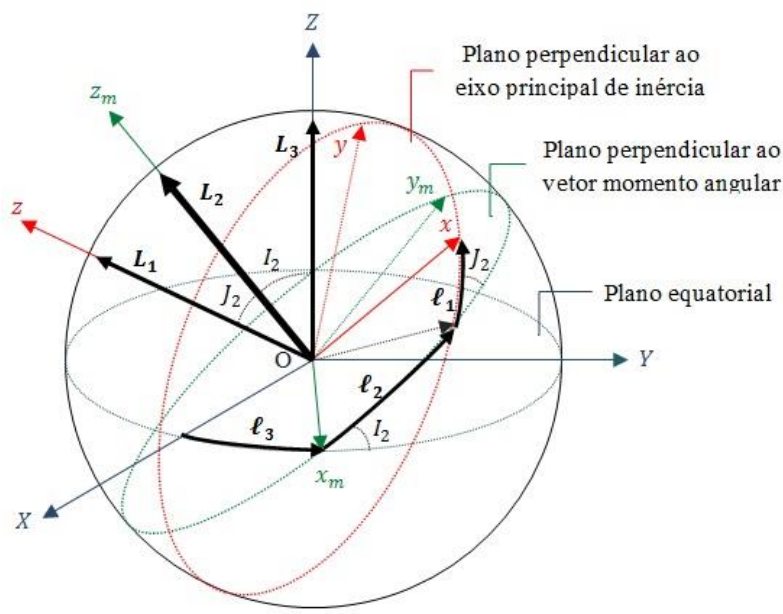

Figura 1 - Variáveis de Andoyer

A Hamiltoniana do movimento rotacional livre de torques externos (MRLTE) para satélites não simétricos (com momentos principais de inércia distintos $A \neq B \neq C$, sendo $A, B, C$ respectivamente os momentos principais em torno dos eixos $\mathrm{x}, \mathrm{y}, \mathrm{z}$ do satélite) é expressa em termos das variáveis de Andoyer por (Kinoshita, 1972) e (Zanardi,1986):

$F=\frac{1}{2}\left[\frac{\sin ^{2} l_{1}}{A}+\frac{\cos ^{2} l_{1}}{B}\right]\left(L_{2}^{2}-L_{1}^{2}\right)+\frac{L_{1}^{2}}{2 C}$

Com as equações do movimento dadas por:

$$
\left\{\begin{array}{c}
\frac{d l_{i}}{d t}=\frac{\partial F}{\partial L_{i}}, \\
\frac{d L_{i}}{d t}=-\frac{\partial F}{\partial \ell_{i}} ;
\end{array} \quad(i=1,2,3)\right.
$$

\section{Equações do Movimento e Pontos de Equilíbrio}

O estudo da estabilidade é feito através dos pontos de equilíbrio, analisando o plano de fase $\left(l_{1}, L_{1}\right)$, sendo que: $0 \leq l_{1} \leq \pi$ e $-L_{2} \leq L_{1} \leq L_{2}$, a partir das equações do movimento (2), pelas quais tem-se:

$$
\begin{aligned}
& \dot{L}_{2}=\dot{L}_{3}=\dot{l}_{3}=0 \\
& \dot{\boldsymbol{l}}_{\mathbf{1}}=-\left(\frac{\sin ^{2} \boldsymbol{l}_{1}}{A}+\frac{\cos ^{2} \boldsymbol{l}_{1}}{\boldsymbol{B}}\right) \boldsymbol{L}_{1}+\frac{\boldsymbol{L}_{1}}{\boldsymbol{C}} \\
& \dot{L}_{1}=-\frac{1}{2}\left(\frac{1}{A}-\frac{1}{B}\right)\left(L_{2}^{2}-L_{1}^{2}\right) \sin 2 l_{1} \\
& \dot{l}_{2}=\left(\frac{\sin ^{2} l_{1}}{A}+\frac{\cos ^{2} l_{1}}{B}\right) L_{2}
\end{aligned}
$$

Observa-se assim, que quando não estão sendo considerados os torques externos, as variáveis métricas $L_{2}$ e $L_{3}$ e a variável angular $l_{3}$ permanecem constantes.

Os pontos de equilíbrio são obtidos das equações de movimento (4), (5) e (6) que resultam em:

$$
\text { 1) } \ell_{1}=0, L_{1}=0
$$

$$
\begin{aligned}
& \text { 2) } \ell_{1}=\frac{\pi}{2}, L_{1}=0 \text {; } \\
& \text { 3) } \ell_{1}=\pi, L_{1}=0 \text {; }
\end{aligned}
$$

Para analisar o espaço de fase $\left(\ell_{1}, L_{1}\right)$ é preciso determinar a Matriz Hessiana da Hamiltoniana do movimento rotacional livre de torques externos que é definida como:

$$
P_{M R L T E}=\left(\begin{array}{cc}
\frac{\partial^{2} F}{\partial \ell_{1}^{2}} & \frac{\partial^{2} F}{\partial \ell_{1} \partial L_{1}} \\
\frac{\partial^{2} F}{\partial L_{1} \partial \ell_{1}} & \frac{\partial^{2} F}{\partial L_{1}^{2}}
\end{array}\right)
$$

de modo que a partir de (1), suas derivadas parciais são expressas como:

$$
\begin{aligned}
& \frac{\partial^{2} F}{\partial \ell_{1}^{2}}=\left(\frac{1}{B}-\frac{1}{A}\right)\left(L_{1}^{2}-L_{2}^{2}\right) \cos 2 \ell_{1} \\
& \frac{\partial^{2} F}{\partial \ell_{1} \partial L_{1}}=\frac{\partial^{2} F}{\partial L_{1} \partial \ell_{1}}=\left(\frac{1}{B}-\frac{1}{A}\right) L_{1} \operatorname{sen} 2 \ell_{1} \\
& \frac{\partial^{2} F}{\partial L_{1}^{2}}=\frac{1}{C}-\frac{\operatorname{sen}^{2} \ell_{1}}{A}-\frac{\cos ^{2} \ell_{1}}{B}
\end{aligned}
$$

A partir do determinante da matriz Hessiana $\left(\Delta=\operatorname{det}\left(\mathrm{P}_{\mathrm{MRLTE}}\right)\right)$ e de seus elementos é possível determinar se os pontos de equilíbrio são um máximo, um mínimo relativo ou ponto de sela (Swokowski, 1995). A análise será aqui realizada considerando a relação $\mathrm{C}<\mathrm{B}<\mathrm{A}$ entre os momentos principais de inércia. Outras análises para os planos de fase $\left(\ell_{1}, L_{1}\right)$ envolvendo outras relações entre os momentos principais de inércia foram realizadas por DEPRIT (1967) e Cabette (2006).

\section{Análise dos Pontos de Equilíbrio para Momentos Principais de Inércia $\mathrm{C}<\mathrm{B}<\mathrm{A}$}

Considerando o caso em que os momentos principais de inércia o momento angular $L_{2}$ e sua projeção $L_{l}$ assumem os seguintes valores:

$$
\begin{gathered}
A=3,94 * 10^{5} \mathrm{Kg} \mathrm{m}^{2} \\
B=3,33 * 10^{5} \mathrm{Kg} \mathrm{m}^{2} \\
C=1,03 * 10^{5} \mathrm{Kg} \mathrm{m}^{2} \\
L_{1}=1 * 10^{4} \mathrm{Kg} \mathrm{m}^{2} / \mathrm{s} \\
L_{2}=9.3707 * 10^{3} \mathrm{Kg} \mathrm{m}^{2} / \mathrm{s}
\end{gathered}
$$

os quais são similares aos dados do satélites americano PEGASUS (Zanardi,1986), pode-se analisar cada ponto de equilíbrio:

1) Para $\ell_{1}=0, L_{1}=0$ e $\ell_{1}=\pi, L_{1}=0$ obtém-se: 
$\frac{\partial^{2} F}{\partial L_{1} \partial \ell_{1}}=\frac{\partial^{2} F}{\partial L_{1} \partial \ell_{1}}=0, \quad \frac{\partial^{2} F}{\partial \ell_{1}^{2}}<0, \quad \frac{\partial^{2} F}{\partial L_{1}^{2}}>0, \quad \Delta<0$

que são definidos como pontos de sela (Swokowski, 1995).

A Hamiltoniana nesses pontos assume 0 seguinte valor

$$
F_{B}=\frac{L_{2}^{2}}{2 B}=141,9780 \mathrm{Kg} \mathrm{m}^{2} / \mathrm{s}^{2}
$$

E corresponde a uma rotação em torno do eixo principal de inércia $\mathrm{Oy}$, caracterizando pontos instáveis.

2) Para $\ell_{1}=\frac{\pi}{2}, L_{1}=0$ obtém-se:

$\frac{\partial^{2} F}{\partial L_{1} \partial \ell_{1}}=\frac{\partial^{2} F}{\partial L_{1} \partial \ell_{1}}=0, \frac{\partial^{2} F}{\partial \ell_{1}^{2}}>0, \frac{\partial^{2} F}{\partial L_{1}^{2}}>0, \Delta>0$

que é definido como um ponto mínimo relativo, caracterizando um ponto de equilíbrio estável na ausência de torques externos. valor

A Hamiltoniana nesse ponto assume o seguinte

$$
F_{A}=\frac{L_{2}{ }^{2}}{2 A}=119,8594 \mathrm{Kg} \mathrm{m}^{2} / \mathrm{s}^{2}
$$

E corresponde a rotação em torno do eixo principal de inércia Ox.

Na Figura 2 é apresentado o comportamento da Hamiltoniana $F$ em função de $\left(l_{1}, L_{1}\right)$, na qual é possível visualizar o ponto de mínimo relativo $\left(l_{1}, L_{1}, F_{A}\right)=\left(\frac{\pi}{2}, 0,119.86\right)$ e os pontos de sela $\left(l_{1}, L_{1}, F_{B}\right)=(0,0,141,98) \mathrm{e}$ $\left(l_{1}, L_{1}, F\right)=(\pi, 0,141.98)$.

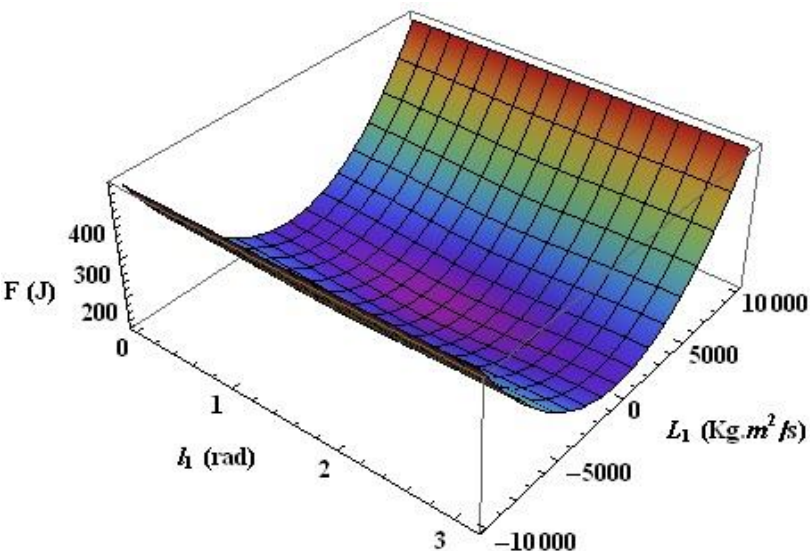

Figura 2 - Comportamento da Hamiltoniana do MRLTE para $\mathrm{C}<\mathrm{B}<\mathrm{A}$ (Silva,2011).

A Figura 3 apresenta o espaço de fase $\left(\ell_{1}, L_{1}\right)$. $\mathrm{Na}$ análise deste plano de fase, observa-se que a Hamitoniana assume valores entre.

$$
\begin{aligned}
& F_{A} \leq F \leq F_{B} \\
& F_{B} \leq F \leq F_{C}
\end{aligned}
$$

onde $F_{C}$ é um caso especial quando $L_{1}= \pm L_{2}$, que corresponde a uma rotação em torno do eixo $\mathrm{Oz}$, com a Hamiltoniana dada por:

$$
F_{C}=\frac{L_{2}{ }^{2}}{2 C}=459,3311 \mathrm{Kg} \mathrm{m}^{2} / \mathrm{s}^{2}
$$

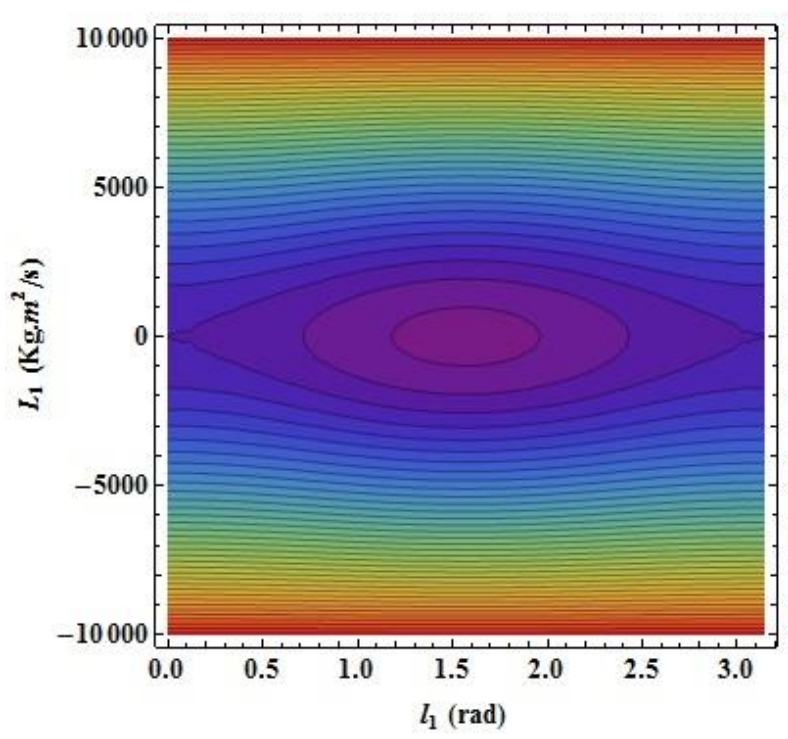

Figura 3- Espaço de fase $\left(\ell_{1}, L_{1}\right)$ para $\mathrm{C}<\mathrm{B}<\mathrm{A}$. $\mathrm{O}$ ponto

$\left(l_{1}, L_{1}\right)=\left(\frac{\pi}{2}, 0\right)$ corresponde à hamiltoniana $F_{A}$, a separatriz corresponde à hamiltoniana $F_{B}$ e a Hamiltoniana $F_{C}$ corresponde ao caso de $L_{1}=L_{2}$

Quando a Hamiltoniana assume valores entre $F_{A}$ e $F_{B}$ ocorrem librações em torno do ponto de equilíbrio estável $\left(l_{1}, L_{1}\right)=\left(\frac{\pi}{2}, 0\right) ;$ a separatriz ocorre quando $F=F_{B}$ e quando a Hamiltoniana assume valores entre $F_{B}$ e $F_{C}$ ocorrem circulações em torno do ponto de equilíbrio estável.

\section{Análise das regiões de circulação}

A seguir são analisados os comportamentos temporais de $l_{1}, l_{2}$ e $L_{1}$ para algumas regiões de circulação, correspondendo à $F_{B} \leq F \leq F_{C}$ para um intervalo de tempo de $500.000 \mathrm{seg}$. São considerados três casos de condições iniciais distintos:

Caso I:

$l_{1}=\frac{\pi}{2} \mathrm{rad} ; l_{2}=\frac{\pi}{2} \mathrm{rad}$ e $L_{1}=2000 \mathrm{kgm}^{2} / \mathrm{s}$

Caso II:

$l_{1}=1 \mathrm{rad} ; l_{2}=\frac{\pi}{2} \mathrm{rad}$ e $L_{1}=2000 \mathrm{kgm}^{2} / \mathrm{s}$

Caso III:

$l_{1}=1 \mathrm{rad} ; l_{2}=\frac{\pi}{2} \mathrm{rad}$ e $L_{1}=7000 \mathrm{kgm}^{2} / \mathrm{s}$ 
As Figuras 4, 5 e 6, apresentam respectivamente o comportamento temporal da variável $l_{1}, l_{2}$ e $L_{1}$ para os casos I, II e III.

Observa-se na Figura 4 um comportamento periódico para a variável $l_{1}$, sendo que o período do caso III é muito maior do que dos casos I e II. Na Figura 5 é mostrada uma variação linear para a variável angular $l_{2}$, com diferenças não significativas entre os três casos. Na Figura 6 observa-se um comportamento periódico da variável $L_{1}$, nos casos I e II, e uma variação menos para o caso III.

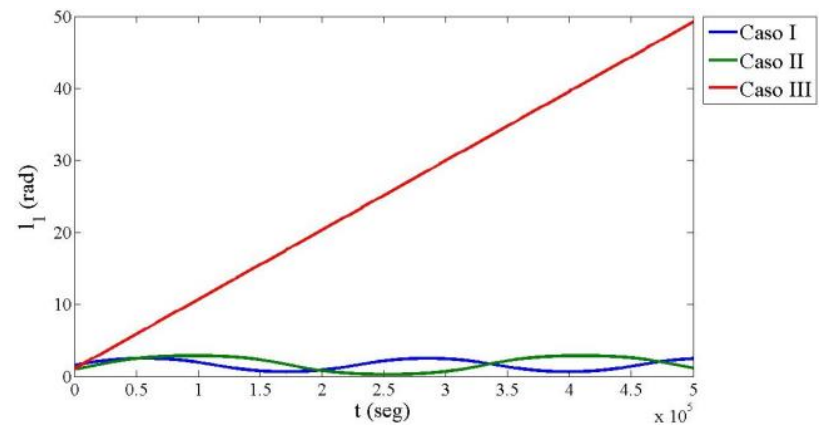

Figura 4 - Comportamento temporal de $l_{1}$, para região de circulação nos casos I, II e III.

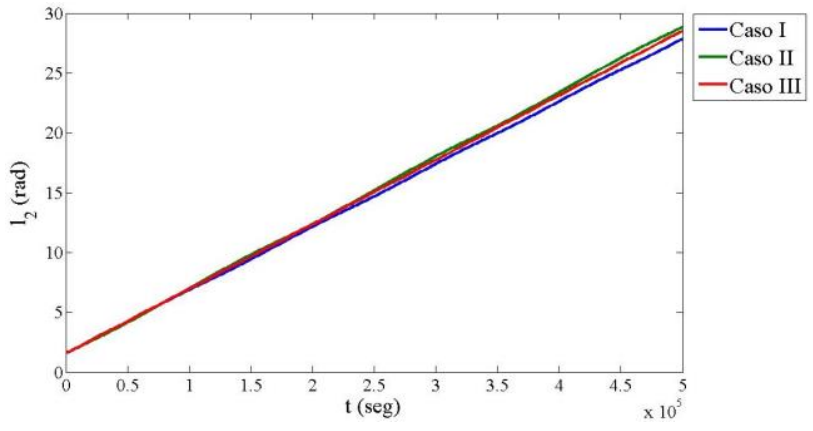

Figura 5 - Comportamento temporal de $l_{2}$, para região de circulação nos casos I, II e III.

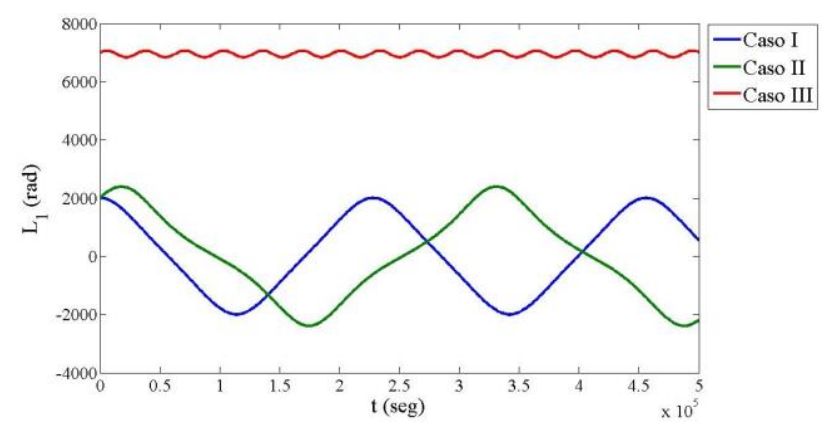

Figura 6 - Comportamento temporal para $L_{1}$ na região de circulação nos casos I, II e III.
Pela Figura 6, observa-se que quando os valores de $L_{l}$ se aproximam do valor de $L_{2}$ (correspondendo a ângulo $J_{2}=0^{\circ}$ ), as amplitudes das variações periódicas diminuem. Tal comportamento é justificado pelo coeficiente do termo periódico na equação do movimento de $L_{l}$, dada pela equação (5).

$\mathrm{Na}$ Figura 4, observam-se pequenas variações periódicas na variável angular $l_{l}$ quando $L_{l}$ é bem menor do que $L_{2}$ (casos I e II) e uma variação linear quando $L_{1}$ se aproxima de $L_{2}$ (caso III). Esta variação linear se justifica pelo fato da pequena variação de $L_{l}$ no caso III, o que acarreta em variação linear em $l_{l}$ (associada ao termo $L_{l} / C$ ) que prevalece sob o outro termo da equação (4).

\section{Análise da região de libração}

A seguir são analisados os comportamentos temporais de $l_{1}, l_{2}$ e $L_{1}$ para algumas regiões de libração, correspondendo à $F_{A} \leq F \leq F_{B}$ para um intervalo de tempo de $500.000 \mathrm{seg}$. São considerados três casos de condições iniciais distintos:

Caso IV:

$l_{1}=\frac{\pi}{2} \mathrm{rad} ; l_{2}=\frac{\pi}{2} \mathrm{rad}$ e $L_{1}=500 \mathrm{kgm}^{2} / \mathrm{s}$

Caso V:

$l_{1}=1 \mathrm{rad} ; l_{2}=\frac{\pi}{2} \mathrm{rad}$ e $L_{1}=1000 \mathrm{kgm}^{2} / \mathrm{s}$

\section{Caso VI:}

$l_{1}=1 \mathrm{rad} ; l_{2}=\frac{\pi}{2} \mathrm{rad}$ e $L_{1}=1500 \mathrm{kgm}^{2} / \mathrm{s}$

Nas Figuras 7, 8 e 9, respectivamente estão apresentados os comportamentos temporais das variáveis $l_{1}, l_{2}$ e $L_{1}$ para os casos IV, V e VI.

Pelas Figuras 7 e 9 observa-se que as variáveis $l_{1}$ e $L_{1}$ assumem um comportamento periódico, não havendo muitas diferenças entre os casos. As amplitudes do movimento aumentam com o aumento de $L_{l}$.

Pela variável $l_{2}$ varia linearmente, também não havendo diferenças significativas entre os casos, como na análise da região de circulação. Isso se justifica pelo fato da região de libração ser muito pequena para este satélite. 


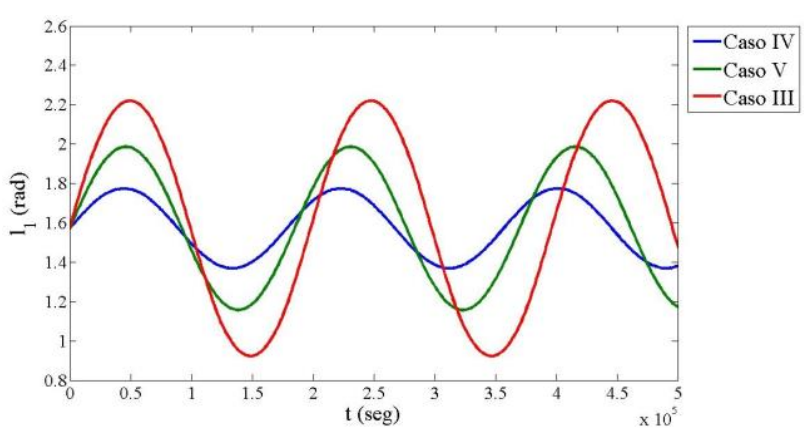

Figura 7 - Comportamento temporal para $l_{1}$ na região de libração nos casos IV, V e VI.

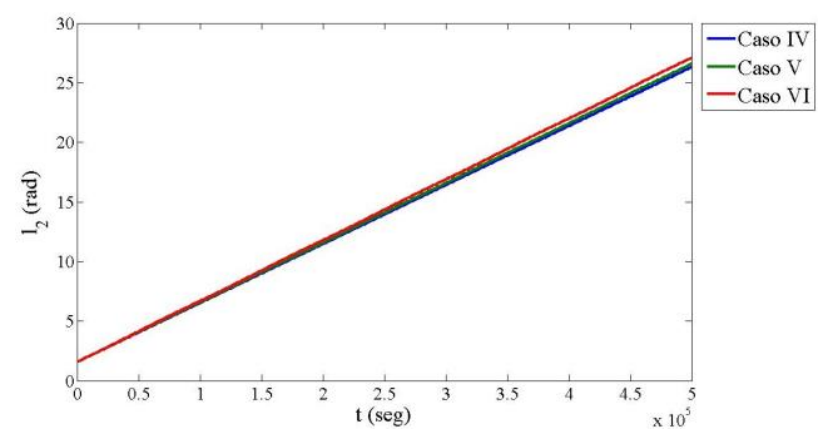

Figura 8-Comportamento temporal para na região de libração nos casos IV, V e VI.

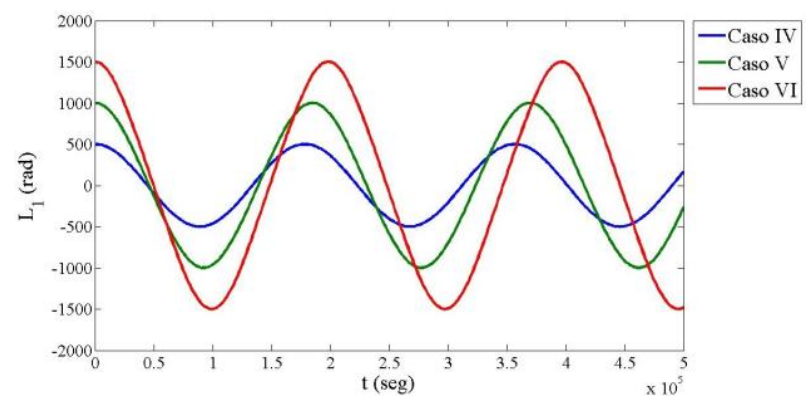

Figura 9-Comportamento temporal para na região de libração nos casos IV, V e VI.

\section{Conclusão}

Neste trabalho foi analisado o movimento rotacional livre de torques externos, descrito pelas variáveis canônicas de Andoyer, com aplicações para um satélite de médio porte com momentos principais de inércia distintos. Pontos de equilíbrio foram determinados, sendo dois pontos instáveis e um ponto estável. O comportamento temporal em regiões de circulação e libração apresentam variações periódicas em uma variável angular e uma métrica e uma variação linear na variável angular diretamente relacionada com a rotação do satélite. Os resultados obtidos são importantes para a continuidade da análise do movimento rotacional quando os torques externos serão incluídos nas equações do movimento.

\section{Agradecimentos}

Os autores agradecem o apoio do $\mathrm{CNPq}$ e da FAPESP (processo no. 2012/21023-6).

\section{Referência Bibliográfica}

Cabette, R. E. S.: "Estabilidade do movimento rotacional de satélites artificiais". Dissertação de doutorado, Instituto Nacional de Pesquisas Espaciais, São José dos Campos, (2006).

Zanardi, M. C.: "Study of the terms of coupling between rotational and translational motion". Celes. Mech. 39(2), 147 - 164, (1986). DOI: 10.1007/BF01230847

Silva, W. R.: "Estudo da estabilidade do movimento rotacional de satélites artificiais com variáveis canônicas". Qualificação de mestrado, Universidade Estadual de São Paulo, Guaratinguetá, (2011)

Deprit, A. Free Rotation of a Rigid Body Studied in the Phase Plane. Am. J. Phys., v.55, 1967

Swokowski, E. W.: Cálculo com Geometria Analítica. v.I. McGraw-Hill, 1995 